
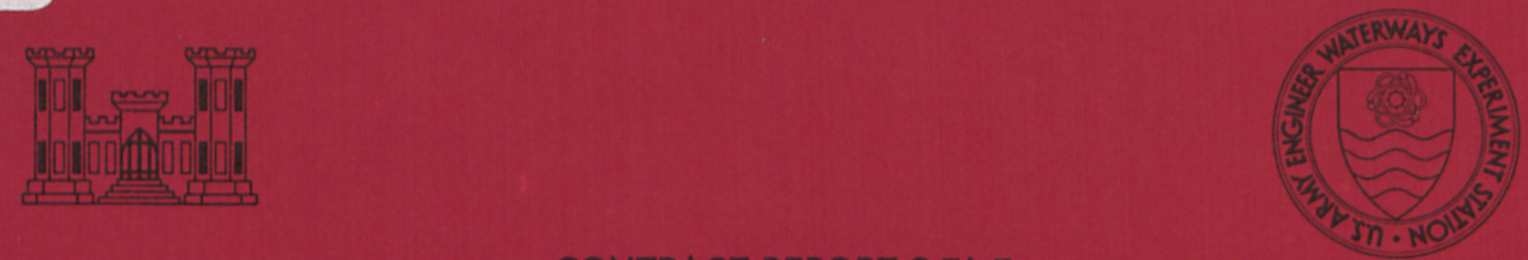

\title{
SATURATED SAND AS AN INELASTIC TWO-PHASE MEDIUM
}

\author{
by \\ Zdenek P. Bazant and Raymond J. Krizek \\ The Technological Institute, Department of Civil Engineering \\ Northwestern University, Evanston, III. 60201
}

August 1976

Final Report

Approved For Public Release; Distribution Unlimited

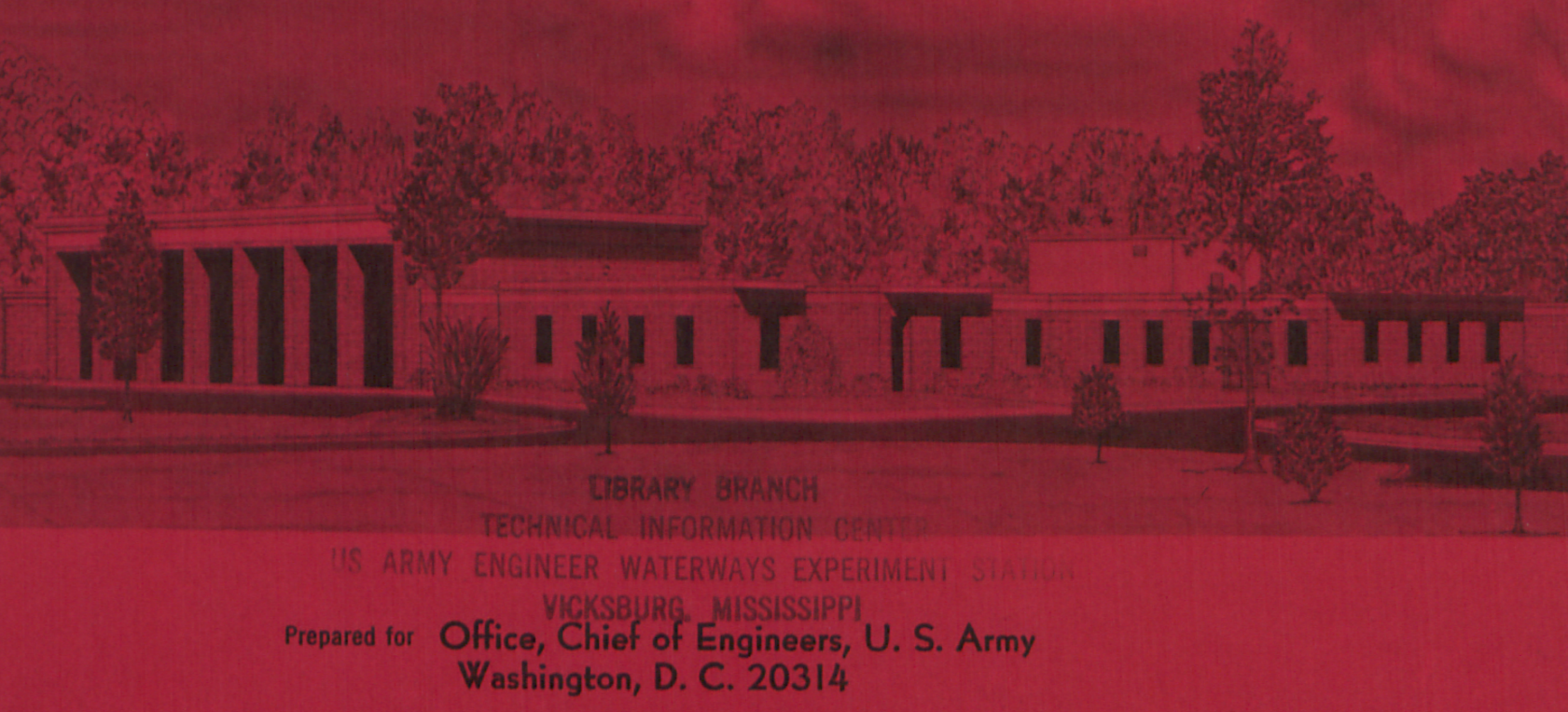

Under Contract No. DACW39-75-M-3739

Monitored by Soils and Pavements Laboratory

U. S. Army Engineer Waterways Experiment Station

P. O. Box 631, Vicksburg, Miss. 39180 


\begin{tabular}{|c|c|}
\hline REPORT DOCUMENTATION PAGE & $\begin{array}{l}\text { READ INSTRUCTIONS } \\
\text { BEFORE COMPLETING FORM }\end{array}$ \\
\hline \begin{tabular}{l|l} 
1. REPORT NUMBER & 2. GOVT ACCESSION NO. \\
Contract Report $5-76-7$ &
\end{tabular} & 3. RECIPIENT'S CATALOG NUMBER \\
\hline $\begin{array}{l}\text { 4. TITLE (and Subtitio) } \\
\text { SATURATED SAND AS AN INELASTIC TWO-PHASE }\end{array}$ & $\begin{array}{l}\text { 5. TYPE OF REPORT \& PERIOD COVERED } \\
\text { Final Report }\end{array}$ \\
\hline MEDIUM & 6. PERFORMING ORG. REPORT NUMBER \\
\hline $\begin{array}{l}\text { 7. AUTHOR(a) } \\
\text { Zdenek P。Bazant } \\
\text { Raymond J。 Krizek }\end{array}$ & $\begin{array}{l}\text { 8. CONTRACT OR GRANT NUMBER(0) } \\
\text { DACW39-75-M-3739 }\end{array}$ \\
\hline $\begin{array}{l}\text { 9. PERFORMING ORGANIZATION NAME AND ADDRESS } \\
\text { The Technological Institute } \\
\text { Department of Civil Engineering } \\
\text { Northwestern University, Evanston, Ill. }\end{array}$ & $\begin{array}{l}\text { 10. PROGRAM ELEMENT, PROJECT, TASK } \\
\text { AREA \& WORK UNIT NUMBERS }\end{array}$ \\
\hline $\begin{array}{l}\text { 11. CONTROLLING OFFICE NAME AND ADDRESS } \\
\text { Office. Chief of Engineers. }\end{array}$ & $\begin{array}{l}\text { 12. REPORT DATE } \\
\text { August } 1976\end{array}$ \\
\hline Washington, D. C. 20314 & $\begin{array}{l}\text { 13. NUMBER OF PAGES } \\
28\end{array}$ \\
\hline $\begin{array}{l}\text { T4. MONITORING AGENCY NAME A ADDRESS(ll difforont from Controllind Offico) } \\
\text { U。 S. Army Engineer Waterways Experiment Station } \\
\text { Soils and Pavements Laboratory }\end{array}$ & $\begin{array}{l}\text { 15. SECURITY CLASS. (of thlo soport) } \\
\text { Unclassified }\end{array}$ \\
\hline P. 0. Box 631, Vicksburg, Miss. 39180 & $\begin{array}{l}\text { 15a. DECLASSIFICATION/DOWNGRADING } \\
\text { SCHEDULE }\end{array}$ \\
\hline
\end{tabular}

16. DISTRIBUTION STATEMENT (of thlo Roport)

Approved for public release; distribution unlimited.

17. DISTRIBUTION STATEMENT (of the abatract ontorod in Block 20, If difforent from Roport)

18. SUPPLEMENTARY NOTES

19. KEY WORDS (Continue on roverce slde If nocessary and Identlly by block number)

Liquefaction (Soils)

Pore water pressure

Sands

Saturated soils

20. ABSTAACT (Continue an roverse atob if necoecery and tdentlfy by block number)

The inelastic densification produced by shear straining saturated sands is opposed by the elasticity of the pore water and leads to a pore pressure increase, which causes a decrease in the intergranular frictional forces and consequent liquefaction of the sand mass. This inelastic densification is accompanied by an inelastic strain of the fluid phase, and the magnitude of the developed pore water pressure is the product of the inelastic densification and the densification compliance, the latter being approximately equal to the drained compressibility of the sand. The tangent (incremental) (Continued) 
20. ABSTRACT (Continued). elastic moduli are expressed in terms of the drained and undrained compressibilities of the two-phase medium and the compressibilities of water and the solid matter forming the grains. It is demonstrated that the volume change of the grains due to intergranular stresses has a negligible effect on the material parameters, even though it roughly equals the volume change of the grains due to the pore water pressure, which has an appreciable effect. Typical values are calculated for the material parameters. 
THE CONTENTS OF THIS REPORT ARE NOT TO BE USED FOR ADVERTISING, PUBLICATION, OR PROMOTIONAL PURPOSES. CITATION OF TRADE NAMES DOES NOT CONSTITUTE AN OFFICIAL ENDORSEMENT OR APPROVAL OF THE USE OF SUCH COMMERCIAL PRODUCTS. 


\section{Preface}

This report was prepared by Professors Zdenek P. Bazant and Raymond J. Krizek of Northwestern University under Contract No. DACW3975-M-3739 as part of ongoing work at the U. S. Army Engineer Waterways Experiment Station (WES) under CWIS 31145 work unit entitled "Liquefaction Potential of Earth Dams and Foundation."

The contract was monitored by Dr. William F. Marcuson III of the Earthquake Engineering and Vibrations Division (EE\&VD), Soils and Pavements Iaboratory (S\&PL). General guidance was provided by Mr. James P. Sale, Chief of S\&PL; Mr. Stanley J. Johnson, Special Assistant to the Chief of S\&PL; and Mr. Walter C. Sherman, Jr., and Dr. Francis G. McLean, former Chief and Chief of EE\&VD, respectively. Contracting Officer was COL G. H. Hilt, CE, Director of WES . 


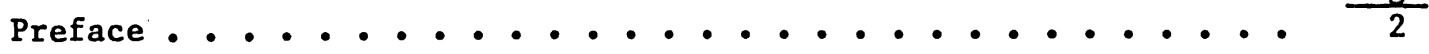

Introduction ......................... . 4

Assumptions . . . . . . . . . . . . . . . . . . . 5

Homogeneity and Isotropy .................. 5

Shear Stresses ........................ 5

Volumetric Deformations ............... 6

Contact Areas ..................... 6

Compressibility of Grains ................. 7

Basic Relattons ..................... 7

Conservation of Mass .................. 7

Stress Resultants ................... 8

Elastic Strains ..................... 9

Definition of Inelastic Strains . . . . . . . . . 10

Incremental Elastic Moduli . . . . . . . . . . . . 12

Auxiliary Relations . . . . . . . . . . . . 12

Bulk Compressibility . . . . . . . . . . . . . 14

Undrained Bulk Compressibility . . . . . . . . . . . 16

Compressibility Due to Pore Fluid Pressure . . . . . . 16

Compression of Dry Sand ................. 18

Calculation of Elastic Moduli from Test Results . . . . . . . 18

Inelastic Strains in Solid and Fluld Phases . . . . . . . . 22

Pore Pressure Due to Densification in Undrained Sand . . . . . 23

Remark on Practical Application . . . . . . . . . . . . 24

Conclusions . . . . . . . . . . . . . . . . . . 24

References . . . . . . . . . . . . . . . . . 25

Appendix A: Pore Water Compressibility with Dilation of

Skeletion at Constant Overburden . . . . . . . . . Al

Appendix B: Notation ...................... B1 


\section{INTRODUCTION}

Inelastic shear strains in saturated sands occur essentially without interference of pore water, but inelastic volumetric strains caused by the rearrangement of grain configurations in the process of shear straining are resisted elastically by the water filling the pores. Therefore, a coupling between the solid skeleton of sand grains and the pore water exists, and a saturated sand must be treated as a two-phase medium. The linear elastic and linear viscoelastic theory of such a medium has been developcd by Biot (1956, 1957), but an extension of this theory into the nonlinear inelastic range is necessary to formulate inelastic densifications. This extension has acquired considerable importance in recent years due to the increascd concern with the dynamic densification and assuciated liquefaction of saturated sand deposits as a result of seismic disturbances.

Despite the recognized need for such a two-phase medium formulation from a theoretical point of view, its practical usefulness requires that the elastic moduli be expressed in terms of easily and directly measurable quantities, such as the compressibility of water $C_{w}$ and the compressibilities $C_{b}$ and $C_{t}$ of saturated sand under drained or undrained conditions. Although studies devoted to the determination of elastic moduli for a two-phase medium have been reported by Blot (1957) and Ishihara (1967), appropriate expressions are not available. Omitting $C_{t}$ and $C_{w}$, Biot (1957) expressed these moduli in terms of the so-called unjacketed compressibilities of the solid skeleton and the pore water due to a pressure applied to the pore water, but these parameters are not particularly useful for practical applisations. In addition, certain small terms (such as porosity increments duc to deformation) were neglected without of fering justification. The derivation of modulus expressions in terms of $C_{b}, C_{t}$, and $C_{w}$ has been attempted by Ishihara (1967), but several of his basic equations are incorrect (for 
example, Equation(9)is correct only in spatial (Euler) coordinates while all remaining equations are written in material (Lagrangian) coordinates, in which case this equation should read $n\left(d_{p}-d_{s}+d e\right)=d_{n}$, and the assumption that precedes Equation (14), as well as the assumption that the volume change of the grains is caused only by pore water pressure, are unjustified). The stress-strain relations considered herein will be expressed in an incremental form, so that the present analysis is applicable to nonlinear behavior that is typical of sands.

\section{ASSUMPTIONS}

Homogeneity and Isotropy

The granular solid (sand) is assumed to be statistically homogeneous on a sufficiently large scale and incrementally isotropic. The assumption of 1sotropy is hardly ever true in the strict sense, even in a sand which is perfectly isotropic in the unstressed state, because the contact areas between grains depend on the stress and those contact areas which are normal to the major principal stress are larger, so that the sand must be stiffer in this direction. Nevertheless, the effect of the associated anisotropy is probably small and negligible. As a consequence of isotropy, the incremental stress-strain relations may be treated as separate relations for the deviatoric and volumetric components of the siress and strain tensors.

\section{Shear Stresses}

The shear stresses are assumed to be carried totally by the solid skeleton (as for dry sands) and the portion of the macroscopic shear stresses carried by the fluid phase is neglected (see Figure 1). Consequently, the incremental stress-strain relations for nonlinear inelastic deviatoric deformations involve only deviatoric stress and strain increments of the solid skeleton and not the fluid phase. 


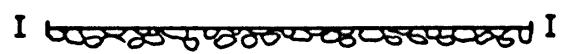

$$
\sigma_{f}=\sigma+\sigma_{F}
$$

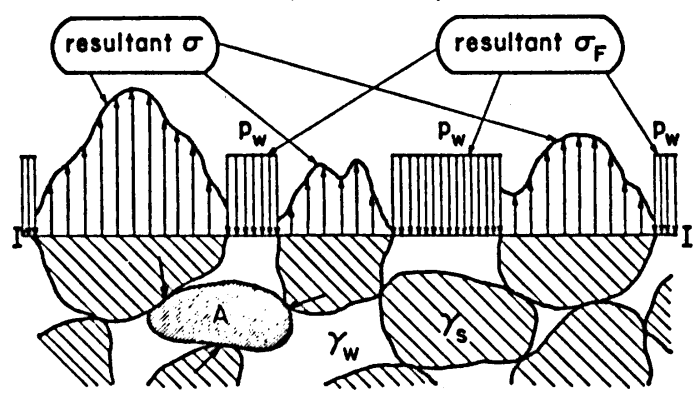

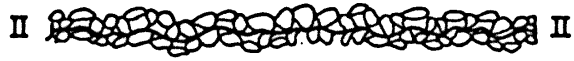

$$
\sigma_{t}=\sigma^{\prime}+\left(-D_{w}\right)
$$

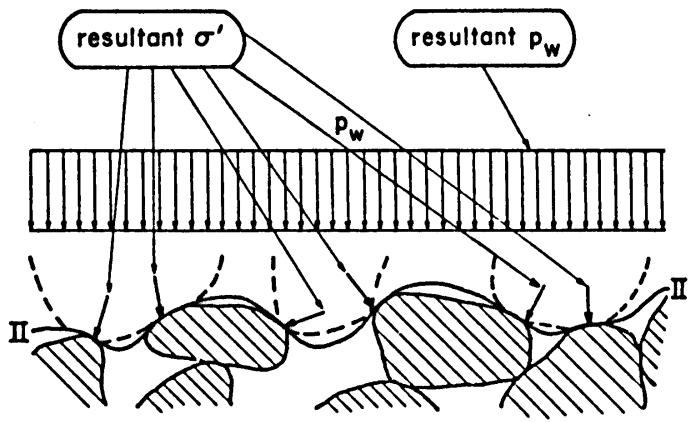

Figure 1. Sketch of Sand Cross section Elucidating Various Macroscopic Stress Components

Volumetric Deformations

The volumetric deformations of the fluid within the pores are assumed to be perfectly elastic and given by

$$
\frac{d \gamma_{w}}{\gamma_{w}}=c_{w} d p_{w}
$$

in which $\gamma_{w}$ is the mass density of the pore water and $p_{w}$ is the fluid pressure, which will be taken as positive in the case of compression. Normal stresses, denoted $\sigma$ (with a subscript of superscript), will be positive for tension.

\section{Contact Areas}

The contact areas between the sand grains are assumed to be negligibly small so that the pore water and associated pressure $\mathrm{p}_{\mathrm{w}}$ completely surrounds each grain. This assumption also precludes the existence of appreciable bond forces between the grains. As a consequence of this assumption, the macroscopic resultant of the intergranular contact forces, called the effective stress $\sigma^{\prime}$, may be determined by considering a macroscopically planar, but microscopically sinuous, unit cross section which passes only through interparticle contacts (Section II-II in Figure 1). On such a 
cross section the pore water pressure $p_{w}$ acts over the entire area and must be subtracted from the total macroscopic volumetric stress $\sigma$ to obtain the resultant of the intergranular forces:

$$
\sigma^{\prime}=\sigma_{t}-\left(-p_{w}\right)
$$

\section{Compressibility of Grains}

Each individual grain is assumed to be perfectly elastic with a bulk compressibility $\mathrm{C}_{\mathrm{S}}$. Since each grain is loaded by pressure $\mathrm{P}_{\mathrm{W}}$ over its entire boundary, there is a uniform hydrostatic pressure $F_{w}$ within the grain, and this causes a volumetric compression $C_{s} p_{w}$ of the grain. In addition, each grain (such as $A$ in Figure 1 ) is also loaded by the intergranular forces which are in excess of $P_{w}$ and characterized precisely and solely by $\sigma^{\prime}$. Thus, the additional volume change is proportional to $\sigma^{\prime}$, so that

$$
\frac{d \gamma_{s}}{\gamma_{s}}=c_{s} d p_{w}-c_{s}^{\prime} d \sigma^{\prime}
$$

in which $\mathrm{C}_{S}^{\prime}$ is the compressibility of grains due to intergranular stress. $C_{S}^{\prime}$ is an average statistical property of the sand, whereas $C_{S}$ and $C_{w}$ are properties of homogeneous substances and are not of a statistical nature. In previous studies, $C_{S}^{\prime}$ has not been taken into account; there is no a priori reason for neglecting $C_{s}^{\prime}$, but it will be seen subsequently in Table 1 that the effect of $C_{s}^{\prime}$ is small compared to $C_{s}$, even when $C_{s}^{\prime}$ do' is larger than $\mathrm{C}_{s} \mathrm{dp}_{\mathrm{w}}$

\section{BASIC RELATIONS}

\section{Conservation of Mass}

The kinematic variables needed to describe the behavior of a two-phase medium are most conveniently chosen as the macroscopic (statistical average) displacements of the solid $u_{k}$ and fluid $U_{k}$, where sub:icript $k(k=1,2,3)$ 
refers to cartesian axes $x_{k}$. The volumetric deformations are then characterized by the volumetric strain of the solid skeleton $\varepsilon=\partial u_{k} / \partial x_{k}=\operatorname{div} u_{k}$ and the volumetric (macroscopic) strain of the pore fluid $\epsilon_{\mathrm{F}}=\partial u_{k} / \partial x_{k}=$ div $U_{k}$, where the subscript $F$ refers to the macroscopic fluid phase. The strains may be expressed with the help of the (macroscopic) bulk mass densities of the solid phase $\rho$ and fluid phase $\rho_{F}$ which are related to $\gamma_{S}$ and $\gamma_{w}$ by

$$
p=(1-n) \gamma_{s}
$$

and

$$
P_{F}=n \gamma_{w}
$$

The condition of conservation of mass yields the mass continuity relations $\dot{\rho}=-\rho$ div $\dot{u}_{k}$ and $\dot{p}_{F}=-\rho_{F}$ div $\dot{U}_{k}$, in which the dots represent material (rather than spatial) time derivatives. From these equations it follows that

$$
d_{\rho}=-\rho d c
$$

and

$$
d p_{F}=-p_{F} d \varepsilon_{F}
$$

Note that the formulation developed herein, including that of the fluid phase, is expressed in terms of material (Lagrangian) coordinates.

\section{Stress Resultants}

The force variables that are associated with $\epsilon$ and $\epsilon_{F}$ by means of a work expression are not $\sigma^{\prime}, \sigma_{t}$, or $P_{w}$, but the macroscopic volumetric stresses in the solid phase $\sigma$ and the fluid phase $\sigma_{F}$ defined such that $\sigma \delta \varepsilon+\sigma_{F} \delta \varepsilon_{F}$ is the correct work expression. The work done by, the fluid flow per unit material element at $\delta \varepsilon=0$ equals $-\mathrm{p}_{\mathrm{w}} \delta \mathrm{V}_{\mathrm{F}}$, where $\delta \mathrm{V}_{\mathrm{F}}=\mathrm{n} \delta \varepsilon_{\mathrm{F}}$ is the volume of water that flows out of the unit element. Thus, $\sigma_{F} \delta \varepsilon_{F}=$ $-p_{w}\left(n \delta \varepsilon_{F}\right)$, which yields

$$
\sigma_{F}=-n p_{w}
$$


as the only possible definition of $\sigma_{F}$. Equation (6) represents the resultant of the pore water pressure $p_{w}$ over a unit cross section of porosity 11 ; this: cross section is perfectly planar (not microscopically sinuous) and pilsses through the grains (Section I-I in Figure 1). The stress in the solid phase $\sigma$. must then represent the resultant of the stresses acting over the remaining area, $1-n$, of the unit cross section (this is equivalent to the resultant of the microscopic stresses in the solids on this crosssection). However, it makes no sense to write for $\sigma$ an expression analogous to Equation (6), because, unlike $p_{w}$, the microscopic stresses in the solids are not uniformly distributed within the grains (unless $\sigma^{\prime}=0$ ). In terms of the stress and strain tensors $\sigma_{i j}$ and $\varepsilon_{i j}$ for the solid phase, $\sigma$ and $\epsilon$ may be written as $\sigma=\frac{1}{3} \cdot \sigma_{k k}$ and $\epsilon=\frac{1}{3} \epsilon_{k k^{\circ}}$. By equilibrium of the macroscopic cross section, the total volumetric stress is $\sigma_{t}=\sigma+\sigma_{F}$, which allows $\sigma^{\prime}$ to be written as

$$
\sigma^{\prime}=\sigma+\sigma_{F}+P_{w}=\sigma+(1-n) p_{w}=\sigma-\frac{1-n}{n} \sigma_{F}
$$

\section{Elastic Strains}

Due to the nonlinearity of sand, the stress-strain relations will be expressed in an incremental form. The elastic strain increments of the two-phase medium $d \epsilon=d \epsilon^{e l}$ and $d \epsilon_{F}=d \epsilon_{F} e^{l}$ are defined as the strain increments that are perfectly reversible upon removal of the stress increment. This implies the existence of an incremental strain energy density, $W$, as a function of $d \varepsilon^{e l}$ and $d \varepsilon_{F}{ }^{e l}$, and since $\Delta \sigma=\partial W / \partial \epsilon=\left(\partial^{2} W / \partial \varepsilon^{2}\right) \Delta \varepsilon+$ $\left(\partial^{2} W / \partial \epsilon \partial \epsilon_{F}\right) \Delta \varepsilon_{F}$ and $\Delta \sigma_{F}=\partial W / \partial \varepsilon_{F}=\left(\partial^{2} W / \partial \epsilon_{F} \partial \epsilon\right) \Delta \varepsilon+\left(\partial^{2} W / \partial \epsilon_{F}^{2}\right) \Delta \varepsilon_{F}$, the volumetric stress-strain relation must have the form (Ishihara, 1967)

$$
\left\{\begin{array}{l}
d \sigma \\
d \sigma_{F}
\end{array}\right\}=\left[\begin{array}{ll}
P & Q \\
Q & R
\end{array}\right]\left\{\begin{array}{l}
d \epsilon^{e l} \\
d \epsilon_{F}^{e l}
\end{array}\right\}
$$


in which $P, Q$, and $R$ are tangent (incromental) volumetric colastic moduli ol the two-phase medium $\left(P=\partial^{2} W / \partial \epsilon^{2} ; Q=\partial^{2} W / \partial \epsilon \partial \epsilon_{p} ; R=\partial^{2} W / \partial \epsilon_{p}^{2}\right)$. H Ho symmetry of this matrix is a consequence of the existence of an incremental potential.

\section{Definition of Inelastic Strains}

In general, the strain increments are composed of reversible and irreversible components, the latter of which are called inelastic strain increments $d e^{\prime \prime}$ and $d \varepsilon_{F}^{\prime \prime}$ and defined as the strains that occur without any change in stress (i.e. at do $=\mathrm{d} \sigma_{F}=0$ ). According to this definition, $d \epsilon^{e l}=d \epsilon-d \epsilon^{\prime \prime}$ and $d \epsilon_{F}^{e l}=d \epsilon_{F}-d \epsilon_{F}^{\prime \prime}$, so that Equation (8) becomes

$$
\mathrm{d} \sigma=P\left(d \epsilon-d \epsilon^{\prime \prime}\right)+Q\left(d \epsilon_{F}-d \epsilon_{F}^{\prime \prime}\right)
$$

and

$$
d \sigma_{F}=Q\left(d \epsilon-d \epsilon^{\prime \prime}\right)+R\left(d \epsilon_{F}-d \epsilon_{F}^{\prime \prime}\right)
$$

The inelastic strain or densification strain of the solid phase de" represents the inelastic densification that results from the slip of grains over each other and the consequent rearrangement of particles into denser configurations (at $d \sigma=0$ ). The strain increment $d \epsilon^{\prime \prime}$ is a functional of the deviatoric strains and stresses in the solid skeleton itself. One of the advantages of postulating a two-phase medium is that $d \epsilon^{\prime \prime}$ may be considered to depend on the shear strains in the same manner as for unsaturated sands. Actually, of course, the same densification can not occur in a saturated sand, but the difference from $d \epsilon^{\prime \prime}$, representing the elastic resistance of the pore fluid against densification, is handled by Equations (1), (3), and (5).

Although the introduction of an inelastic strain in the fluid phase might seem illogical, the fluid phase should not be confused with the fluid (water) 1tself. In general, the fluid phase displacements $U_{k}$ may result 
from both the volume compression of the fluid (which is perfectly elastic by virtue of Equation (1)) and the change in pore space (which may be inelastic) due to the slip of grains over each other and the consequent rearrangement of particles into denser configurations. At this point the possibility that $d \varepsilon_{F}^{\prime \prime}=0$ is not excluded, but it will be shown later that this is impossible, because the inelastic densification of the solid phase (de at do $=0$ ) is accompanied by a contraction of the pore space, and this can not occur at $\mathrm{d} \sigma_{\mathrm{F}}=0$ (or $\mathrm{d} \gamma_{\mathrm{W}} \approx 0$ ) unless water is expelled from the pore space. I'o expel water, a divergent llux is gencrated (i.a. div $\|_{k}=0$ ) which means that $d \epsilon_{F} \neq 0$ at $d \sigma_{F}=d \sigma=0$. The notion of an inclastic strain in the fluid phase and the necessity of its dependence on $d \epsilon^{\prime \prime}$ were originally suggested by Z.J. Bazant (1967) without establishing the proper relationship for $\mathrm{d} \epsilon_{\mathrm{F}}^{\prime \prime} / \mathrm{d} \epsilon^{\prime \prime}$, as given subsequently in Equation (43).

The change of state of saturated sand is fully specified by $d \epsilon, d \epsilon_{F}$, and $d \varepsilon_{F}^{\prime \prime}$. Thus, since Equations $(1),(3),(4),(5),(6),(7)$, and (9) represent a system of 10 equations, it should be possible to determine the 10 unknowns $\left(d \sigma, d \sigma_{F}, d \varepsilon_{F}^{\prime \prime}, d \sigma^{\prime}, d p_{w}, d n, d \gamma_{w}, d \gamma_{s}, d \rho\right.$, and $\left.d \rho_{F}\right)$ when $d \epsilon$, $d \epsilon_{F}$, and $d \epsilon^{\prime \prime}$ are given. If $d \epsilon_{F}^{\prime \prime}$ were omitted, there would be only 9 unknowns versus 10 equations to be satisfied, and the problem would then be unsolvable. Hence, it is seen that $d e_{\mathrm{F}}^{\prime \prime}$ must be included in Equation (9), and the solution of the 10 equations will relate $d \varepsilon_{F}^{\prime \prime}$ to $d \varepsilon^{\prime \prime}$. The establishment of this relationship will be one objective of the subsequent analysis. Another objective will be the determination of $P, Q$, and $R$ in terms of readily measurable or known quantities, such as $\mathrm{C}_{w}, \mathrm{C}_{s}$, and $\mathrm{n}$. 


\section{Auxiliary Relations}

To develop relationships for the incremental moduli, it is expedient to first eliminate some of the variables. Differentiation of liquations (4) gives $d \rho=(1-n) d \gamma_{s}-\gamma_{s} d n$ and $d \rho_{F}=n d \gamma_{w}+\gamma_{w} d n$, which, upon substitution for $d \rho$ and $d \rho_{F}$ from Equations (5) and for $\rho$ and $\rho_{F}$ from Equations (4), allows $\rho$ and $\rho_{s}$ to be eliminated, this yielding

$$
d \varepsilon=\frac{d n}{1-n}-\frac{d \gamma_{s}}{\gamma_{s}}
$$

and

$$
d \epsilon_{F}=-\frac{d n}{n}-\frac{d \gamma_{w}}{\gamma_{w}}
$$

The further use of Equations (1) and (3) to eliminate $\gamma_{s}$ and $\gamma_{w}$ gives

$$
d \epsilon_{0}=\frac{d n}{1-n}-C_{s} d p_{w}+C^{\prime} d \sigma^{\prime}
$$

and

$$
d \varepsilon_{F}=-\frac{d n}{n}-C_{w} d p_{w}
$$

Differentiation of Equation (6) and substitution for $d p_{w}$ from Equation

yields $d \sigma_{F}=-p_{w} d n-n d \gamma_{w} / \gamma_{w} C_{w}$, which, upon replacement of $d \gamma_{w}$ from

Equation $(10 b)$, can be written as $d n=\left(C_{w} d \sigma_{F}-n d \varepsilon_{F}\right) /\left(1-C_{w} p_{w}\right)$. Inserting

this expression for dn into Equation (11a), together with $d p_{w}=\sigma_{F} d n / n^{2}-d \sigma_{F} / n$ and $d \sigma^{\prime}=d \sigma-(1-n) d \sigma_{F} / n+\sigma_{F} d n / n^{2}$ from Equations (7) and (6), gives

$$
C_{1} d \sigma_{F}+(1-n) C_{S}^{\prime} d \sigma=(1-n) d \varepsilon+n \beta d \epsilon_{F}
$$

in which

$$
\begin{aligned}
& \beta=\frac{1+\frac{1-n}{n}\left(C_{s}-C_{s}^{\prime}\right) p_{w}}{1-C_{w} p_{w}} \\
& C_{1}=C_{0}-\frac{(1-n)^{2}}{n} C_{s}^{\prime}
\end{aligned}
$$




$$
c_{0}=B C_{w}+\frac{1-n}{n} C_{s}
$$

In most cases of practical interest, $P_{w} \leq 0.5 \mathrm{~N} / \mathrm{mm}^{2}$, which corresponds to a depth of less than $50 \mathrm{~m}$ below the surface. The use of this value for $P_{w}$, together with typical values for $C_{w}$ and $C_{s}\left(C_{w}=0.49 \mathrm{~mm}^{2} / \mathrm{kN} ; c_{s}=0.028 \mathrm{~mm}^{2} / \mathrm{kN}\right)$ gives $C_{w} p_{w} \leq 3 \times 10^{-4}$ and $C_{s} p_{w}(1-n) / n \leq 3 \times 10^{-5}$, which indicates that all terms with $p_{w}$ are negligible and $\beta \doteq 1$.

Substituting for do from Equation (9) and considering the special case of elastic deformations ( $d \epsilon^{\prime \prime}=d \epsilon_{F}^{\prime \prime}=0$ ) leads to

$$
d \sigma_{F}=\frac{1}{C_{1}}\left\{(1-n)\left(1-C_{S}^{\prime} P\right) d \varepsilon+\left[\beta n-(1-n) C_{S}^{\prime} Q\right] d \varepsilon_{F}\right.
$$

which holds for any values of $d \varepsilon$ and $d \varepsilon_{F}$; Since the coefficients of $d \varepsilon$ and $d \varepsilon_{F}$ must be the same as those in Equations (9), $Q$ and $R$ may be written as

$$
Q=\frac{1-n}{C_{1}}\left(1-C_{s}^{\prime} P\right)
$$

and

$$
R=\frac{\beta n-(1-n) C_{s}^{\prime} Q}{C_{1}}
$$

The basic equations will now be expressed in terms of directly measurable quantities, such as $p_{w}$ or $\sigma^{\prime}$ instead of $\sigma$ and $\sigma_{F}$. Equations (7) and (6) yield $d \sigma=p_{w} d n-(1-n) d p_{w}+d \sigma^{\prime}$ and $d \sigma_{F}=-n d p_{w}-p_{w} d n$, respectively, and the substitution for $d n$ and $d e_{F}$ from Equations (11) and the use of Equations (9) with $d \epsilon^{\prime \prime}$ and $d \varepsilon_{F}^{\prime \prime}=0$ gives

$$
\mathrm{d} \sigma=d \sigma^{\prime}-(1-n) d p_{w}-P_{w} n\left(d \varepsilon_{F}+C_{w} d p_{w}\right)=P d \varepsilon+Q d \varepsilon_{F}
$$

and

$$
d \sigma_{F}=P_{w} n\left(d \epsilon_{F}+C_{w} d p_{w}\right)-n d p_{w}=Q d \epsilon+R d \epsilon_{F}
$$

Upon elimination of $d \varepsilon_{F}$, it follows for elastic deformations that 


$$
\begin{aligned}
\left(R+n p_{w}\right) d \sigma^{\prime}+ & \left\{n Q-(1-n) R-\left[C_{w}(Q+R)-1\right] n P_{w}\right\} d p_{w} \\
& =\left[P R-Q^{2}-(P+Q) n p_{w}\right] d \epsilon
\end{aligned}
$$

The mass of water which is expelled from a unit volune of sand is $\mathrm{dm}=\rho_{\mathrm{F}} \operatorname{div}\left(d U_{k}-d u_{k}\right)=p_{F}\left(d \epsilon_{F}-d \epsilon\right)$, where $\rho_{F}$ is the bulk mass density of the fluid phase. Expressing $d \epsilon_{F}$ from Equation (9) for the elastic case gives $\mathrm{d} \epsilon_{\mathrm{F}}=\left(\mathrm{d} \sigma_{\mathrm{F}}-\mathrm{Qd} \varepsilon\right) / \mathrm{R}$, and replacement of $\mathrm{d} \sigma_{\mathrm{F}}$ yields $\mathrm{d} \epsilon_{\mathrm{F}}=-\left(\mathrm{p}_{\mathrm{w}} \mathrm{dn}+\right.$ $\left.n d p_{w}+Q d \varepsilon\right) / R$. Then, substituting $d n=-n\left(d \epsilon_{F}+C_{w} d p_{w}\right)$ from Equation (11b), provides

$$
\frac{d m}{\gamma_{w}}=n\left(d \epsilon_{F}-d \varepsilon\right)=\frac{n\left(Q-R+n P_{w}\right) d \varepsilon-\left(1-C_{w} p_{w}\right) n^{2} d p_{w}}{R-n P_{w}}
$$

\section{Bulk Compressibility}

Consider a drained compression test in which a saturated soil sample is encased within an impermeable, but flexible, membrane, and the pore water pressure $\mathrm{p}_{\mathrm{w}}$ is maintained constant (i.e. $d \mathrm{p}_{\mathrm{w}}=0$ ). In most tests of this type $\mathrm{P}_{\mathrm{w}}=0$, and all subsequent relations simplify. The load is applied on the external surface of the membrane as a fluid pressure $p_{e}$ which exceeds the constant pore water pressure $P_{w}$ by a value denoted as $p^{\prime}$ and balances the total stress $\sigma_{t}$ in the specimen (i.e. $\sigma_{t}=-p_{e}=-p^{\prime}-p_{w}$ ). Consequently, the external excess fluid pressure $p^{\prime}$ represents the negative intergranular or effective stress $-\sigma^{\prime}$, not the stress in the solid phase $-\sigma$, as assumed in Equation (5) by Biot (1957).

According to Equation (17) for $d p_{w}=0$, the bulk compressibility of the solid skeleton in the presence of fluid in the pores is

$$
c_{b}=\left[\frac{\partial \epsilon}{\partial \sigma^{\prime}}\right]_{P_{w}}=\left[\frac{\partial e}{\partial \sigma_{t}}\right]_{P_{w}}=\frac{R+n p_{w}}{P R-Q^{2}-(P+Q) n P_{w}}
$$

For typical values of $n$ and for $p_{W} \leq 0.5 \mathrm{~N} / \mathrm{mm}^{2}, n p_{W} \leq 0.0003 R$ and $(P+Q) n P_{W} \leq 0.002\left(P R-Q^{2}\right)$; hence, the terms containing $P_{W}$ can be neglected, 
and Equation (19) reduces to

$$
C_{b}=\frac{R}{P R-Q^{2}}
$$

Although it is quite reasonable to neglect the indicated terms in problems involving relatively shallow depths (less than $50 \mathrm{~m}$ ) of natural sand, these terms may be important in situations involving very largo depths or highly compressible granular materials which are much different from natural sands.

Another expression for $C_{b}$ can be obtained as follows. Expressing $\mathrm{dn}=-\mathrm{nd} \epsilon_{\mathrm{F}}$ from Equation $(10 \mathrm{~b})$ and substituting into Equation (9a) for $\mathrm{d} \epsilon^{\prime \prime} .=\mathrm{d} \epsilon_{F}^{\prime \prime}=0$ gives $\mathrm{d} \sigma=\mathrm{Pd} \epsilon-\mathrm{Qdn} / \mathrm{n}$. Insertion of $\mathrm{d} \sigma=\mathrm{d} \sigma^{\prime}+\mathrm{P}_{\mathrm{w}} \mathrm{dn}$, which follows from Equation (7) for $\mathrm{dp}_{\mathrm{w}}=0$, yields $\mathrm{d} \sigma^{\prime}=\mathrm{Pd} \epsilon-\left(Q / \mathrm{n}+\mathrm{p}_{\mathrm{w}}\right) \mathrm{dn}$, and further substitution of $d n=(1-n)\left(d \varepsilon-C_{s}^{\prime} d \sigma^{\prime}\right)$ from Equation (11a) leads to $d \sigma^{\prime}=P d \epsilon-\left(Q / n+P_{w}\right)(1-n)\left(d \epsilon-C_{s}^{\prime} d \sigma^{\prime}\right)$. This may be rewritten as $d \epsilon=C_{b} d \sigma^{\prime}$, in which

$$
C_{b}=\frac{n-(1-n)\left(Q+n p_{w}\right) C_{s}^{\prime}}{n P-(1-n)\left(Q+n p_{w}\right)}
$$

Since $n p_{w} \leq 0.0003 Q$ for typical values of $n$ and for $p_{w} \leq 0.5 \mathrm{~N} / \mathrm{mm}^{2}$, Equation (21) reduces to

$$
c_{b}=\frac{n-(1-n) Q C_{s}^{\prime}}{n P-(1-n) Q}
$$

According to Equation (18) the mass of water expelled from the pores of a unit volume of sand is characterized by the coefficient

$$
w_{b}=\frac{1}{Y_{w}}\left[\frac{\partial m}{\partial \varepsilon}\right]_{p_{w}}=n \frac{Q-R+n p_{w}}{R-n p_{w}}
$$

which, for small values of $p_{w}$, simplifies to

$$
w_{b}=n\left(\frac{Q}{R}-1\right)
$$


To determine the undrained bulk compressibility, a saturated sand specimen is enclosed within an impermeable, but flexible, membrane and loaded externally with no drainage allowed. The displacement of the solid and the water are assumed equal at the specimen-membrane interface, and, because of the assumed homogeneity of the specimen, they are equal everywhere (1.e. $u_{k}=U_{k}$ in this type of test). Thus, div $u_{k}=\operatorname{div} u_{k}$ and $d \epsilon=d \epsilon_{k}$, and from Equation (1) for the elastic component of stress it follows that $d \sigma_{t}=d \sigma+d \sigma_{F}=(P+2 Q+R) d a$. Hence, the bulk of total compressibility of the undrained material can be expressed as

$$
C_{t}=\left[\frac{\partial e}{\partial \sigma_{t}}\right]_{\epsilon-\varepsilon_{F}}=\frac{1}{P+2 Q+R}
$$

Since the pore water pressure $P_{w}$ can be measured in an undrained test, Equation (16b) yields

$$
Q_{p}=-\left[\frac{\partial P_{w}}{\partial \epsilon}\right]_{\epsilon-\epsilon_{F}}=\frac{Q+R-n P_{w}}{n\left(1-C_{w} P_{w}\right)}
$$

for $d \epsilon-d \epsilon_{F}=0$. For small values of $P_{w}$, Fquation (26) simplifies to

$$
Q_{p}=\frac{Q+R}{n}
$$

Note that the strain in the solid due to a change in the pore water pressure $\partial \varepsilon / \partial p_{w}$ at a condition of no flow $\left(\varepsilon=\varepsilon_{F}\right)$ equals $1 / Q_{p}$.

\section{Compressibility Due to Pore Fluid Pressure}

To determine the compressibility due to pore fluid pressure, a saturated sand specimen is enclosed within an impermeable, but flexible, nembrane, and loaded with an external cell pressure $P_{e}$ and an internal back pressure $P_{w}$ ouch that $P_{e}-P_{w}=-\sigma^{\prime}=$ constant; although it is possible to cunduct such a test, the resulting deformations would probably be too small to measure accurately. Nevertheless, since d $\sigma^{\prime}=0$ for such a test, Lquation (17) yields 


$$
C_{P}=-\left[\frac{\partial \epsilon}{\partial p_{w}}\right]_{\sigma^{\prime}}=\frac{\dot{n Q}-(1-n) R-\left[(Q+R) C_{w}-1\right] n p_{w}}{P R-Q^{2}-(P+Q) n p_{w}}
$$

which for small values of $P_{w}$ reduces $L$

$$
C_{P}=\frac{n Q-(1-n) R}{P R-Q^{2}}
$$

An alternative expression may be obtalned by differentiating Equations (4a) and (4b), substituting for $d p$ and $d p_{F}$ from Equation (5a) and (5b) and for $d \gamma_{w}$ and $d y_{s}$ from Equations (1) and (3), and eliminating dn from the resuiting two equations; this provides

$$
(1-n) d \epsilon+n d \epsilon_{F}+\left[n C_{w}+(1-n) C_{s}\right] d p_{w}+(1-n) C_{s}^{\prime} d \sigma^{\prime}=0
$$

which, upon setting do' $=0$ and substituting for dep, from liquat fon (l(b)), gives

$$
C_{p}=-\left[\frac{\partial \varepsilon}{\partial p_{w}}\right]_{\sigma^{\prime}}=\frac{n^{2}-R\left[n C_{w}+(1-n) C_{s}\right]+n C_{s} p_{w}}{(1-n) R-n Q-n(1-n) p_{w}}
$$

For small values of $p_{w}$, Equation (31) simplifies to

$$
C_{p}=\frac{n^{2}-R\left[n C_{w}+(1-n) C_{s}\right]}{(1-n) R-n Q}
$$

Similarly, the alternative use of Equation (16a) instead of Equation (16b) gives

$$
C_{p}=\frac{n(1-n)-Q\left[n C_{w}+(1-n) C_{s}\right]}{(1-n) Q-n P}
$$

for small values of $P_{w}$. The mass of water that is forced into a unit volume of sand at $d \sigma^{\prime}=0$ is a quantity that can be measured and is characterized, according to Equation (18), by the coefficient

$$
C_{p w}^{\prime}=-\frac{1}{\gamma_{w}}\left[\frac{\partial m}{\partial p_{w}}\right]_{\sigma^{\prime}}=\frac{n^{2}\left(1-C_{w} p_{w}\right)}{R-n p_{w}}
$$

which for small values of $p_{w}$ becomes 


$$
C_{p w}^{\prime}=\frac{n^{2}}{R}
$$

In a different type of test a sand specimen can be placed in a perfectly rigid container $(d \epsilon=0)$ and the mass of water $m$ that is forced into the container under pressure $\mathrm{p}_{\mathrm{w}}$ can be measured. In this case we obtain the coefficient $C_{p w}^{\prime \prime}=-\left(\partial m / \partial p_{w}\right) / Y_{w}$, which can be easily determined from Equation (18).

Compression of Dry Sand

In the case of dry sand $Q=R=0$ and

$$
P=3 K=3 / C
$$

where $\mathrm{K}$ is the bulk modulus of the solid skeleton and $\mathrm{C}$ is its bulk conpressibility, which, in general, may be different from $C_{b}$. This value may be applied to the two-phase medium, provided the absence of water from grain contacts does not change appreciably the material properties.

\section{CALCULATION OF ELASTIC MODULI FROM TEST RESULTS}

By means of Equations (15), (20), (22), (24), (25), (27), (29), (32), (33), and (35), coefficients $P, Q, R, C_{w}, C_{s}$, and $C_{s}^{\prime}$ are related to various measurable test parameters. Most convenient for measurenent are probably $C_{b}$ and $C_{t}$, and advantage can be taken of the fact that $C_{w}$ is quite accurately known without testing $\left(0.49 \mathrm{~mm}^{2} / \mathrm{kN}\right.$ at $\left.25^{\circ} \mathrm{C}\right)$ and that $\mathrm{C}_{s}$ is approximately known when the mineralogical composition of grains is identified (typically, $c_{s}=0.028 \mathrm{~mm}^{2} / \mathrm{kN}$ for quartz). Furthermore, it is not necessary to know $C_{s}$ too accurately, because its value has only a minor effect on the calculated values of $P, Q$, and $R$.

A method for calculating $P, Q$, and $R$ from $C_{b}, C_{t}, C_{w}, C_{s}$, and $n$ will now be given. Solving Equation (22) for $P$ and substituting the result into Equation (15a) gives a linear algebraic equation for $Q$, which, when combined with Equation (15b) $(\beta=1)$ and Equation (22), yièlds 


$$
\begin{aligned}
& Q=\left(\frac{C_{1}}{1-n} \frac{C_{b}}{C_{b}-C_{s}^{\prime}}+\frac{1-n}{n} G_{s}^{\prime}\right)^{-1} \\
& R=\frac{n-(1-n) C_{s}^{\prime} Q}{C_{1}} \\
& P=\frac{1}{C_{b}}+\frac{1-n}{n} \frac{C_{b}-C_{s}^{\prime}}{C_{b}} Q
\end{aligned}
$$

in which $c_{1}=c_{w}+c_{s}(1-n) / n-c_{s}^{-1}(1-n)^{2} / n$, according to Eqs. (13b) and (13c) These expressions may be evaluated if a value of $\mathrm{C}_{S}^{\prime}$ is assumed. The resulting values must satisfy Equation (25), and so

$$
F\left(C_{s}^{\prime}\right)=0=P+2 Q+R-\frac{1}{C_{t}}
$$

in which $C_{t}$ is an experimentally determined value. Although Equation (38) w111, in general, not be satisfied for an arbitrary value of $C_{s}^{\prime}$, it may be regarded as a function $F\left(C_{S}^{\prime}\right)$ of the chosen $C_{s}^{\prime}$ value and the iterative regula falsi method may be used to find the $C_{s}^{\prime}$ value which gives $F\left(C_{S}^{\prime}\right)=0$.

The required calculations may be shortened by deducing a suitable Initial estimate of $\mathrm{C}_{S}^{\prime}$. Because of equilibrium in the unit cross section of sand, the statistical average $\hat{o}$ of the volumetric component of the microscopic stresses produced within the grains by the intergranular stress $\sigma^{\prime}$ equals $\sigma^{\prime} /(1-n)$. The corresponding relative change in the volume of the grains is $C_{s} \hat{\sigma}$ or $C_{s} \sigma^{\prime} /(1-n)$, and this must equal $C_{s}^{\prime} \sigma^{\prime}$, as indicated by Equation ( 3 ); hence, $C_{s}^{\prime}$ may be estimated by

$$
\mathrm{C}_{8}^{\prime} \approx \frac{\mathrm{C}_{\mathrm{s}}}{1-\mathrm{n}}
$$

which further implies that $C_{1} \approx C_{w}$. Equation (39) also allows the approximate evaluation of $P, Q$, and $R$ from Ẹquations (37) when only $C_{b}$ (and not $C_{t}$ ) has been measured.

The compressibility of the solid material $\mathrm{C}_{s}$ is always much less than 
$\mathrm{C}_{\mathrm{b}}$. For this reason, it may be assumed for a crude approximation that $C_{s} \approx 0$ and $C_{s}^{\prime} \approx 0$. Substituting these values into Equations (15) and (20) yields

$$
\begin{aligned}
& P=\frac{1}{C_{b}}+\frac{Q^{2}}{R} \\
& Q \approx \frac{1-n}{C_{w}} \\
& R \approx \frac{n}{C_{w}}
\end{aligned}
$$

An alternative method of calculation may be based onl liqual ions (2l) and (25). Eliminating $P$ from these two relations gives a quildratic equation for Q, and the only positive solution of this equation is

$$
Q=-R+\left[R\left(\frac{1}{C_{b}}-\frac{1}{C_{t}}\right)\right]^{\frac{1}{2}}
$$

Furthermore, eliminating $C_{S}^{\prime}$ from Equations (15a) and (15b) (in which $C_{1}$ also contains $\left.C_{S}^{\prime}\right)$ and substituting for $P$ from Equation (25) leads to

$$
0=F(R)=\frac{(1-n)^{2}}{n} R-2 Q+(Q+2 R) Q C_{0}+\left(n-R C_{0}\right)\left(\frac{1}{C_{t}}-R\right)
$$

in which $C_{0}=C_{w}+C_{S}(1-n) / n$ and $\beta=1$. Obviously, if Equation (41) were substituted here, a fourth degree algebraic equation for $F$ would result and It would be necessary to solve for $R$ numerically. After insertion of Equation (41), Equation (42) represents a function, $F(R)$, which must be made equal to zero, and the solution for $R$ may be found by the regula falsi method. For the initial estimate of $R$, Equation ( $40 \mathrm{c}$ ) may be used. This method of solution does not involve $C_{s}^{\prime}$, which may be subsequently found, e.g., from Equation (22).

Equations (24), (27), (29), (33), and (35) represent redundant relations, which can be used when $w_{b}, Q_{p}, C_{p}$, and $C_{p w}^{\prime}$ have been measured. They can be employed as a check, and statistical averaging may be performed to reduce 
the experimental error. Equations (32) or (33) may be used to calculate a value for $\left[\mathrm{nC}_{\mathrm{w}}+(1-\mathrm{n}) \mathrm{C}_{\mathrm{s}}\right]$, which allows a check on the assumed values of $C_{w}$ and $C_{S}$. Note that Equation (20) assures that $P R-Q^{2}>0$ because $R>0$ and $c_{b}>0$. Hence, the matrix of elastic constants in Equation (8) is positive definite, which guarantees local stability of the material.

Table 1. Material Parameters of Typical Sands

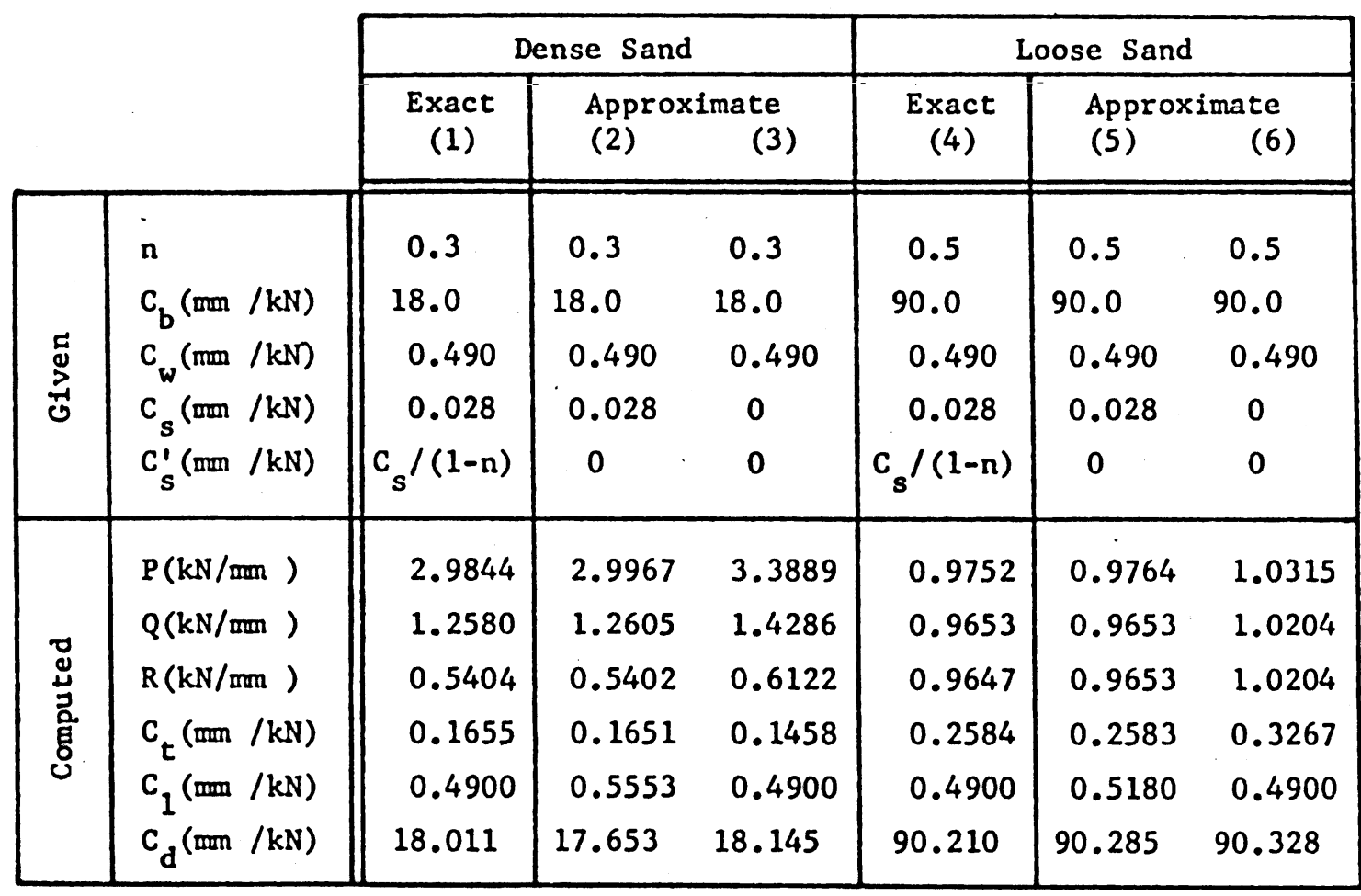

Note: Columns (1), (2), (4), and (5) are calculated from Equations (37), (25), and (50), and columns (3) and (6) from Equations $(40),(25)$, and (51).

The same typical properties of natural sands as those considered by Ishihara (1967) have been used to calculate elastic moduli by both exact and approximate formulas, and the results are given in Table 1. It is scen that, although the value of $C_{S}^{\prime}$ has a negligible effect and may be set equal to either zero or the value given by Eq. (39), C $\mathrm{C}_{\mathbf{s}}$ has a more profound effect. Four decimal places are given in Table 1 only to indicate the order of magnitude of the differences between varlous methods of calculation. From a practical point of view, of course, an error of less than $1 \%$ is meaningless because 
of the uncertainty involved in determining values for the measured properties $\left(e \cdot g \cdot, c_{b}\right)$.

\section{INEIASTIC STRAINS IN SOIID AND VLLUII) PILSI:S}

I'he inclastle strains are dofincd ats Lhose slrains whleh oceur $\mathrm{ul}$ $d \sigma=d \sigma_{F}=0$. Then, Equations $(9 a)$ and $(9 b)$ yield $d t=d \varepsilon^{\prime \prime}$ and $d \varepsilon_{p}=d \varepsilon_{p}^{\prime \prime}$, which, when substituted into Equation (12), give

$$
d \varepsilon_{-F}^{\prime \prime}=-\frac{1-n}{n} d \varepsilon^{\prime \prime}
$$

since $\beta=1$ for small values of $p_{w}$. Perhaps surprisingly, however, the inelastic strain in the fluid phase can not be taken as zero. Equation (43) can also be derived in a more direct, though less rigorous, way by realizing that the changes in unit weights $\gamma_{w}$ and $\gamma_{s}$ must be second-order small quantities when $d \sigma=d \sigma_{F}=0$. Since mass densities do not cliange appreciably, the strain increment $d \epsilon=d \epsilon^{\prime \prime}$ causes the pore volume of the material to expand by $d n=d \epsilon=d \varepsilon^{\prime \prime}$. On the other hand, because the density of the pore fluid does not change appreciably, the volume of the pore water which is imbibed into the material is $n\left(d \epsilon-d \varepsilon_{F}\right)$ or $n\left(d \epsilon^{\prime \prime}-d \varepsilon_{F}^{\prime \prime}\right)$. This must equal $d n$, and so $d n=n\left(d \epsilon^{\prime \prime}-d \epsilon_{F}^{\prime \prime}\right)=d \epsilon^{\prime \prime}$, which yields Equation (43). With the use of Equation (43), Equations (9a) and (9b) reduce to the form

$$
d \sigma=P d \epsilon+Q d \varepsilon_{F}-\left(P-\frac{1-n}{n} Q\right) d \epsilon^{\prime \prime}
$$

and

$$
d \sigma_{F}=Q d \epsilon+R d \epsilon_{F}-\left(Q-\frac{1-n}{n} R\right) d \epsilon^{\prime \prime}
$$

For the typical values of $P$ and $Q$ listed in Table 1 , the quantities $\left(P-\frac{1-n}{n} Q\right)$ and $\left(Q-\frac{1-n}{n} R\right)$ equal $49.0 \mathrm{~N} / \mathrm{mm}^{2}$ and $2.8 \mathrm{~N} / \mathrm{mnn}^{2}$ for $n=0.3$, and $10.5 \mathrm{~N} / \mathrm{mm}^{2}$ and $0.6 \mathrm{~N} / \mathrm{mm}^{2}$ for $\mathrm{n}=0.5$, respectively. Hence, as a rough approximation, $\left(Q-\frac{1-n}{n} R\right) \approx 0$, (i.e. the inelastic strain in Equation (44b) for the fluid phase is negligible) and Equations (44) may be written as 


$$
d \sigma \approx P d \epsilon+Q d \varepsilon_{F}-\frac{1}{C_{b}} d \varepsilon^{\prime \prime}
$$

and

$$
\mathrm{d} \sigma_{\mathrm{F}} \approx \mathrm{Q} \mathrm{d} \epsilon+\mathrm{R} \mathrm{d} \epsilon_{\mathrm{F}}
$$

PORE PRESSURE DUE TO DENSIFICATION IN UNDRAINED SAND

Consider now the sand to be in an undrained condition ( $d \epsilon=d \epsilon_{F}$ ) and let the total stress be constant $\left(d \sigma_{t}=0\right)$, as, for example, when the overburden of a given sand element is constant. Summing liquations (9a) and (9b) and noting that $P+2 Q+R=1 / C_{t}$ (Equation $(25)$ ) and $Q+R \approx 1 / C_{w}$ (liquations (40b) and $(40 c))$, we get

$$
d \sigma_{F}=d \sigma+d \sigma_{F}=\frac{1}{C_{t}} d \epsilon+\left(\frac{Q+R}{n}-\frac{1}{C_{t}}\right) d \epsilon^{\prime \prime}
$$

According to Equations ( $9 \mathrm{~b})$ and (43), the stress produced in the fluid phase by densification is

$$
d \sigma_{F}=Q\left(d \epsilon-d \epsilon^{\prime \prime}\right)+R\left(d \epsilon_{F}-d \epsilon_{F}^{\prime \prime}\right)=\frac{1}{n}\left[R-C_{t}(Q+R)^{2}\right] d \epsilon^{\prime \prime}
$$

The change in the pore water pressure is $d P_{w}=-d\left(\sigma_{F} / n\right)=-d \sigma_{F} / n-P_{w} d n / n=$ - $d \sigma_{F} / n-p_{w}\left(d \varepsilon_{F}+c_{w} d p_{w}\right)$. Since $c_{w} p_{w}<0.0001$ for $p_{w} \leq 0.5 \mathrm{~N} / \mathrm{mm}^{2}$ and since $\mathrm{p}_{\mathrm{w}} \mathrm{de}$ is a second-order small quantity, one has $d \mathrm{p}_{\mathrm{w}} \approx-\mathrm{d} \sigma_{\mathrm{F}} / \mathrm{n}$, and so

$$
d p_{w}=-d \epsilon^{\prime \prime} / C_{d}
$$

in which $C_{d}$, termed the densification compliance, is given by

$$
\frac{1}{C_{d}}=-\left[\frac{\partial P_{w}}{\partial \varepsilon^{\prime \prime}}\right]_{\sigma_{t}, \epsilon-\varepsilon_{F}}=\frac{R-C_{t}(Q+R)^{2}}{n^{2}}
$$

For the case where $c_{s}^{\prime}=0, c_{d}=c_{b}^{2} /\left(c_{b}-c_{t}\right)$. Since the total stress $\sigma_{t}$ remains constant, the build-up of pore water pressure duc to inelastic densification produces, according to Equation (2), a drop in the effective sitress. $d \sigma^{\prime}=-d p_{w}=d \varepsilon^{\prime \prime} / C_{d}$, which leads to a reduction and ultimately the complete loss of the friction forces between grains. This is the underlying cause 
behind the dynamic liquefaction of sand subjected to cyclic shear. A rough estimate of the magnitude of $C_{d}$ may be obtained by putting $1 / C_{t} \approx 1 / C_{b}+1 / n C_{w}$, $R=n / C_{w}$, and $Q+R \approx 1 / C_{w}$, which follows from Equations $(40)$ for $C_{s} \approx C_{s}^{\prime} \approx 0$; Equation (49) then gives

$$
c_{d} \approx\left(\frac{1}{c_{b}}+\frac{1}{n C_{w}}-\frac{1}{n C_{w}}\right)^{-1}\left(1+\frac{n C_{w}}{C_{b}}\right) \approx c_{b}
$$

because normally $\mathrm{C}_{\mathrm{w}} \ll \mathrm{C}_{\mathrm{b}}$. From the typical values of $\mathrm{C}_{\mathrm{a}}$ indicated in Table 1 it is seen that this estimate is quite good; $1 / \mathrm{C}_{\mathrm{d}}$ is orders of magnitude less than $1 / C_{w}$

It is Instructive to note that a much smaller coefficient $\partial p_{w} / \partial \epsilon^{\prime \prime}$ would be obtained, $1 f$, instead of $d \sigma_{t}=0$, the condition were imposed that no elastic strain of the solid accompanies the densification (i.e. de $=\mathrm{d} \epsilon^{\prime \prime}$ ). In this hypothetical case Equation (47) yields $d \sigma_{F}=R\left(d:^{\prime \prime}-d \epsilon_{F}^{\prime \prime}\right) \approx(R / n) d \epsilon^{\prime \prime}$, and substituting for $R$ from Equation $(40), d \sigma_{F} \approx d \varepsilon^{\prime \prime} / C_{w} w d p_{w}=-C_{d}^{\prime} d \varepsilon^{\prime \prime}$, where $C_{d}^{\prime}=-\left[\frac{\partial P_{w}}{\partial \epsilon^{\prime \prime}}\right]_{\epsilon} e_{, \epsilon-\epsilon_{F}}=1 /\left(n C_{w}\right) \gg 1 / C_{b} \approx C_{d}$. However, to enforce the condition that $d_{\epsilon}=d \epsilon^{\prime \prime}$, an enormous change in the total stress $\sigma_{t}$ which can be evaluated from Equation (46), would be necessary.

\section{REMARK ON PRACTICAL APPLICATION}

The ultimate goal of this work is primarily the development of a procedure for predicting the liquefaction of undrained saturated sands subjected to dynamic excitations, such as earthquakes or blasts. However, before this objective can be achieved, the law relating the Inelastic densification, d $\epsilon^{\prime \prime}$, to the history of deviatoric strains must be established.

\section{CONCLUSIONS}

Within the context of this study the following conclusions can be advanced:

1. The Inelastic densification of the solid skeleton de is accompanicd by an inelastic strain in the fluid phase $d e_{F}^{\prime \prime}$ which is related to 
de" (Equation (43)). The fluid itself is perfectly elastic, and $e_{F}^{\prime \prime}$ represents the $f$ low of water that is necessary to allow densification without change in stress.

2. The pore water pressure that is produced in an undrained element of saturated sand by a unit densification at constant total stress is characterized by the densification compliance $C_{d}$, the value of which is approximately equal to the drained compressibility of the solid skeleton $C_{b}$ and is orders of magnitude higher than the compressibility of water $C_{w}$.

3. The tangent (incremental) elastic moduli of a two-phase granular material can be expressed in terms of the drained compressibility $c_{b}$ and the undrained compressibility $C_{t}$ of the medium and known values for the compressibilities of water and the solid matter forming the grains (Equations (37) and (38)).

4. The volume change of the grains due to intergranular stresses (characterized by $C_{s}^{\prime}$ ) has a negligible effect on the properties of a sand, even though it may be of the same order of magnitude as the volume change of the grains due to pore water pressure (characterized by $\mathrm{C}_{\mathrm{s}}$ ), which has an appreciable effect on the elastic moduli of the two-phase medium.

\section{REFERENCES}

1. Bažant, Z. J. (1967), "Theory of Vibration Compaction of Saturated Granular Medium," Proceedings of the International Symposium on Wave Propagation and Dynamic Properties of Earth Materials, University of New Mexico Press, Albuquerque, Ncw Mexico, pp. 767-758.

2. Biot, M. A. (1956), "Theory of Propagation of lilastic Waves in FluidSaturated Porous Solid-I. Low Frequency Range," Journal of the Acoustical Society of America, Volume 28, pp. 168-178.

3. Blot, M. A. (1957), "The Elastic Coefficients of the Theory of Consolidation," Journal of Applied Mechanics, Volume 24, pp. 594-601.

4. Ishihara K. (1967), "Propagation of Compressional Waves in a Saturated Soll," Proceedings of the International Symposium on Wave Propagation and Dynamic Properties of Earth Materials, University of New Mexico Press, Albuquerque, New Mexico, pp. 451-467. 
APPENDIX A

PORE WATER COMPRESSIBILITY WITH DILATION

OF SKFLFTON AT CONSTANI OVERBURDL:N

As is well known, the relation between the diffusivity and permeability of a porous medium involves the compressibility of the pore fluid, which is water in this case. In certain practical situations (e.g. when the overburden of a given sand element remains constant) changes in pore water pressure occur at approximately a constant value of total stress $\sigma_{t}$. Thus, it is of interest to determine the ratio of a change in water content to a change in pore water pressure at $d \sigma_{t}=0$. Adding Equations (9) for $d \epsilon^{\prime \prime}=d \epsilon_{F}^{\prime \prime}=0$, one gets $\mathrm{d} \sigma_{t}=0=(P+Q) \mathrm{d} \epsilon+(Q+R) d \epsilon_{F}$ or $d \epsilon=-d \epsilon_{F}(Q+R) /(P+Q)$, the latter of which may be substituted into Equation $(9 b)$, together with $d \sigma_{F}=-n d p_{w}-p_{w} d n$ and $d n=-n\left(d \epsilon_{F}+C_{w} d p_{w}\right)$ resulting from Equation (11b), to yield $\mathrm{d}_{\mathrm{F}}=\mathrm{n}\left(1-\mathrm{C}_{\mathrm{w}} \mathrm{p}_{\mathrm{w}}\right) \mathrm{d} \mathrm{p}_{\mathrm{w}} /\left[\mathrm{Q}(\mathrm{Q}+\mathrm{R}) /(\mathrm{P}+\mathrm{Q})-\mathrm{R}+\mathrm{np}_{\mathrm{w}}\right]$ at $\mathrm{d} \sigma_{\mathrm{t}}=0$. Equation gives $d m / \gamma_{w}=n\left(d \epsilon_{F}-d \epsilon\right)=n[1-(Q+R) /(P+Q)] d \varepsilon_{F}$, which, upon substitution for $d \epsilon_{F}$, allows $C_{p w}$ to be written as

$$
C_{P w}=-\frac{1}{Y_{w}}\left[\frac{\partial m}{\partial p_{w}}\right]_{\sigma_{t}}=\frac{n^{2}(P-R)\left(1-p_{w} C_{w}\right)}{P R-Q^{2}-n P_{w}(P+Q)} \doteq \frac{n^{2}(P-R)}{P R-Q^{2}}
$$

Furthermore, using the approximations from Equation (40), one gets

$$
c_{p w} \approx(1-2 n) c_{b}+{ }_{n} C_{w} \approx(1-2 n) c_{b}
$$

The compressibility given by Equation (A-2) for constant total stress can be orders of magnitude different from that given by Equation (35) for constant effective stress. For sufficiently large porosities ( $>0.5$ ), $\mathrm{C}_{\mathrm{pw}}$ becones negative; this means that the application of pressure on the pore water does not cause flow into the pore space, but rather out of it, because the solid 
skeleton is dilated by $P_{w}$. Although the physical implications of this phenomenon require further investigation, it can be intuitively understood by noting that $\mathrm{C}_{\mathrm{pw}}$ is a sum of 3 terms: (a) $\mathrm{nC}_{\mathrm{w}}$, which represents the compression of the pore water alone, (b) $c_{b}$, which represents the increase in pore water content due to dilation of the solid skeleton, and (c) $-2 \mathrm{nC}_{b}$, a negative term which represents the deformation of the solid skeleton necessary to produce a stress in the solid phase that offsets the decrease in $\sigma_{t}$ due to $\mathrm{dp}_{\mathrm{w}}$ (i.c. the stress required to maintain $\sigma_{l}$ at its original value). The compressibility $\mathrm{C}_{\mathrm{pw}}$ can be measured by enclosing a specimen in a flexible, impermeable membrane and varying the internal back-pressure $\mathrm{P}_{\mathrm{w}}$ while maintaining the external cell pressure constant. By a similar procedure it can be shown that the strain in the solid due to a change in the pore water pressure at constant overburden is given by

$$
C_{b p}=\left[\frac{\partial \epsilon}{\partial p_{w}}\right]_{\sigma_{t}}=\frac{n(Q+R)\left(1-P_{w} C_{w}\right)}{P R-Q^{2}-n_{w}(P+Q)} \doteq \frac{n(Q+R)}{P R-Q^{2}}
$$

which, upon using the approximations of Equation (40), roduces to

$$
c_{b p} \approx c_{b}
$$




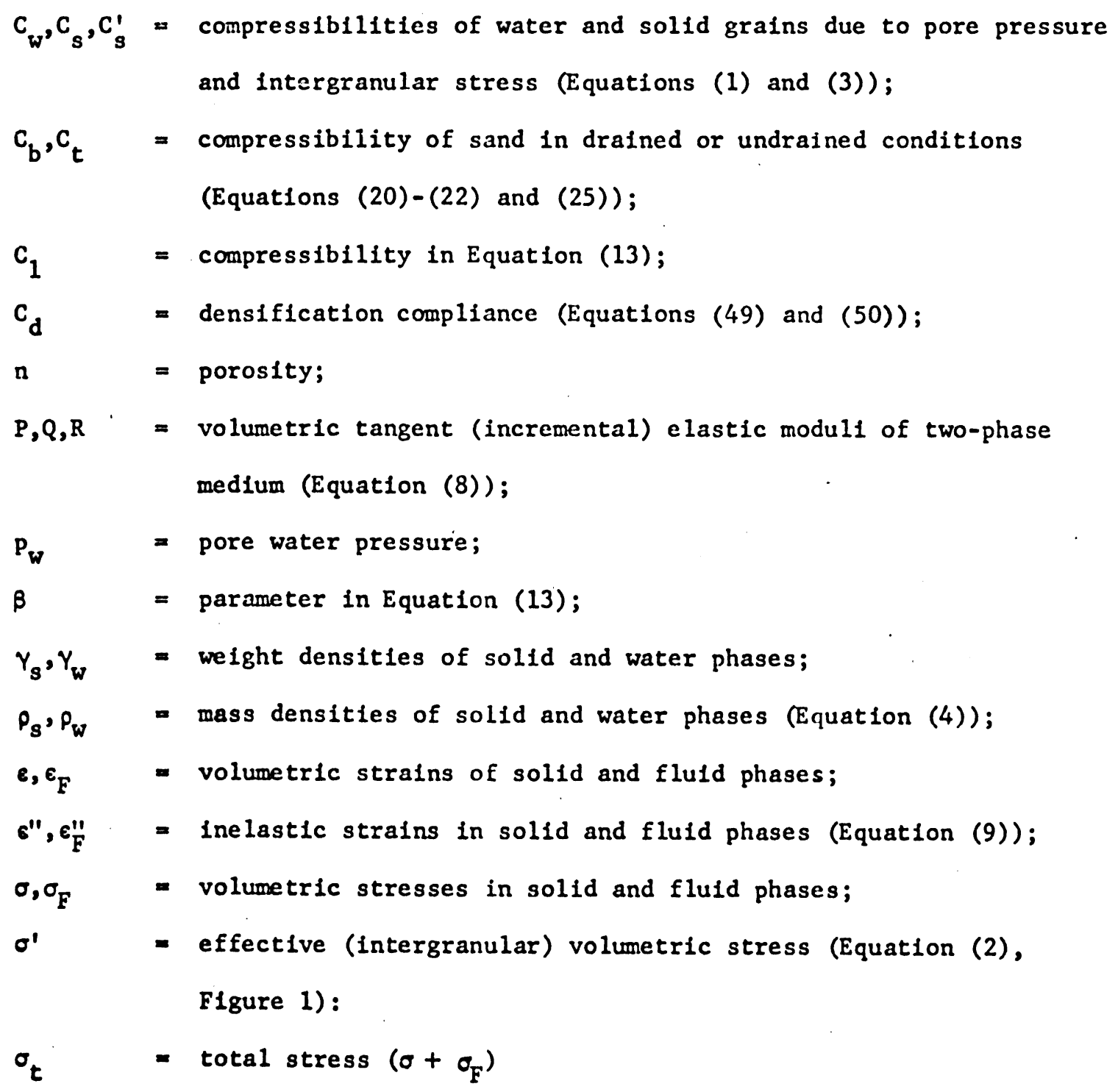

